\section{Autosomal dominant} polycystic kidney disease

Chern Li Chow, Specialist Registrar and Honorary Research Fellow in Nephrology; Albert CM Ong, Professor of Renal Medicine

Academic Unit of Nephrology, School of Medicine, University of Sheffield

Autosomal dominant polycystic kidney disease (ADPKD) is an inherited disease with a prevalence of 1:400 to 1:1,000 live births. ${ }^{1}$ It is the most common genetic cause of renal failure, accounting for $10 \%$ of patients on dialysis. ADPKD is a systemic disorder characterised by progressive kidney enlargement, cyst formation in other organs (liver, pancreas) and non-cystic complications (arterial aneurysm).

\section{Genetics and pathophysiology}

Inheritance of ADPKD is autosomal dominant with $100 \%$ disease penetrance. Each offspring has a $50 \%$ chance of inheriting the disease. There is a 5\% rate of new mutation. Mutations in either of two genes coding for membrane proteins can lead to ADPKD: ${ }^{1}$

- PKD1 (chromosome 1613.3), 85-90\% of cases, encodes for the polycystin-1 protein

- PKD2 (chromosome 4q21), 10-15\% of cases, encodes for the polycystin-2 protein.

A small number of families unlinked to either gene could indicate the potential existence of a third gene (PKD3). PKD1 and PKD2 gene mutations have similar clinical phenotype, but PKD2 patients have a more favourable course. The mean age of onset of end-stage renal failure (ESRF) is 53 years for PKD1 and 69 years for PKD2. Unlike PKD1, women with PKD2 have a later onset of ESRF than men.

\section{Natural history}

Patients are usually asymptomatic until the middle decades, but $2-5 \%$ present in childhood with significant morbidity. By the age of $60,50 \%$ of patients with ADPKD will require renal replacement therapy. Poor prognostic indicators for renal survival include male sex, black race, haematuria before age 30 , multiple pregnancies, hypertension before age 35 , proteinuria, renal size and PKD1 mutation. ADPKD patients do not have a higher risk of mortality than other patients with ESRF. The main cause of mortality is cardiovascular disease $(36 \%$ of cases). ${ }^{2}$

\section{Clinical features}

\section{Pain}

- Renal: acute pain due to infection, stones, intracystic haemorrhage and urinary tract obstruction; chronic pain due to pressure, stretching of the renal capsule or structural distortion.

- Non-renal: liver and pancreatic enlargement or infection.

\section{Hypertension}

Hypertension is a common early finding in $60 \%$ of patients with normal renal function.

\section{Urinary tract infection}

About $30-50 \%$ of patients will have an episode of urinary tract infection in their lifetime.

\section{Cyst haemorrhage and haematuria}

Gross haematuria occurs in $30-50 \%$ of ADPKD patients, with rising incidence as kidney size increases. It usually occurs spontaneously but may be related to physical activity, trauma, stones, infection or tumour.

\section{Nephrolithiasis}

Nephrolithiasis occurs in $20-30 \%$ of patients. The presence of renal calculi should be considered if acute pain, acute deterioration in renal function and haematuria are present.

\section{Massive polycystic kidneys and large renal cysts}

Abdominal discomfort or pain can be caused by massive cysts. Renal cysts are not unique to ADPKD. Other potential causes are given in Table 1.

\section{Cancer}

There is no evidence for an increased risk of renal cell carcinoma in the ADPKD population, but haematuria and flank pain with anorexia and weight loss should prompt further investigation.

\section{Liver cysts}

Hepatic cysts are the most common extrarenal manifestation, increasing with older age $58 \%$ in the third decade and $94 \%$ in the fourth decade on magnetic resonance imaging (MRI). ${ }^{3}$ They occur more frequently and severely in females, correlating with oestrogen exposure (eg pregnancy, contraceptive pill). ${ }^{4}$ They are usually asymptomatic and do not lead to hepatic failure. Pain from compression, feeling of satiety, cystic haemorrhage and infection are the most common symptoms. Rarely, massive polycystic liver disease (female preponderance) can result in portal hypertension. ${ }^{3}$ Rare cases of congenital hepatic fibrosis have also been described. ${ }^{5}$

\section{Intracerebral aneurysms}

Intracerebral aneurysms (ICA) occur in $6 \%$ of ADPKD patients without a family history of ICA and in $16 \%$ of patients with a positive family history of ICA. ${ }^{6}$ However, the most common neurological event in ADPKD is hypertensive haemorrhage or ischaemic stroke.

\section{Symptoms of chronic kidney disease}

Lethargy, poor appetite, reduced urine volume, fluid retention etc are all symptoms of chronic kidney disease (CKD). 


\section{Pancreatic cysts}

Although pancreatic cysts occur in $9 \%$ of patients older than 20 years, this is not related to pancreatic dysfunction. ${ }^{7}$

Table 1. Renal cystic diseases.

\section{Genetic}

Autosomal dominant:

- ADPKD

- Tuberous sclerosis

- Von Hippel-Lindau disease

- Medullary cystic disease

- RCAD

Autosomal recessive:

- ARPKD

- Juvenile-onset nephronophthisis

- Other rare syndromes associated with multiple malformationse

X-linked:

- Oral-facial-digital syndrome type 1

Chromosomal disorders:

- Trisomy 21

- Trisomy 13

- Trisomy 18

- Trisomy C

\section{Investigation}

Table 2 lists the investigations used in assessing ADPKD patients.

New unified diagnostic ultrasound criteria for at-risk PKD1 and PKD2 indi-

ADPKD = autosomal dominant polycystic kidney disease; ARPKD = autosomal recessive polycystic kidney disease; RCAD = renal cysts and diabetes syndrome.

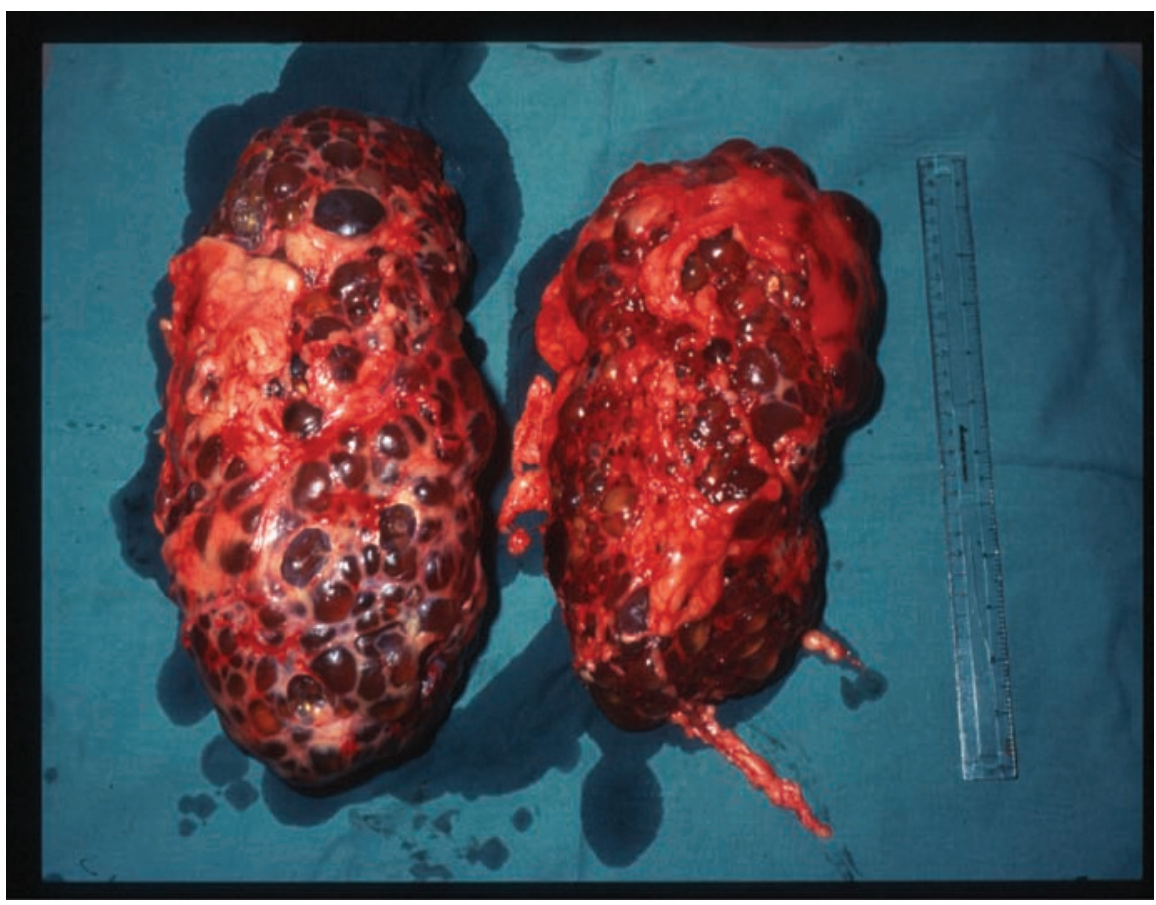

Fig 1. End-stage autosomal dominant polycystic kidneys.

viduals have recently been reported (Table 2). ${ }^{8}$ The previously used criteria had a sensitivity of $100 \%$ in PKD1 patients aged 30 years or older but false-negative rates for $\mathrm{PKD} 2$ (23\%) and $\mathrm{PKD} 1$ (5\%) in younger patients. Where the family history is unknown or negative, these new criteria may not apply and mutation analysis may be indicated for either disease exclusion or diagnostic purposes. ${ }^{8,9}$ Clinically, the presence of bilateral enlarged cystic kidneys with hepatic cysts and absence of positive extrarenal manifestations (suggesting an alternative cystic disease) make the diagnosis highly probable.

The Consortium for Radiologic Imaging Studies of PKD (CRISP) suggests that the rate of disease progression can be monitored through serial MRI kidney volume measurements prior to significant decline in glomerular filtration rate. ${ }^{10}$

\section{Management}

There is no cure for ADPKD. Treatment is aimed at blood pressure control, prompt treatment of infections and disease complications, and the management of CKD.

\section{Pain}

Pain should be treated with simple analgesia. Lifestyle modification and avoidance of aggravating activities can also be useful. ${ }^{3}$ Patients may require pain clinic referral and surgical management (cyst decompression, renal denervation or nephrectomy).

\section{Hypertension}

The aim is to achieve a blood pressure below 130/80 mmHg. ${ }^{15}$ There is no specific evidence that any particular antihypertensive drug is more effective in ADPKD. A trial currently in progress, Halt Progression in PKD (HALT-PKD), is investigating whether the use of angiotensin-converting enzyme inhibitors or angiotensin receptor blockers confers additional benefit.

\section{Urinary tract infection}

Urine cultures may be persistently negative in cyst infection. Ureteric obstruction from stone or clot with associated infection 
should be excluded. Patients will require prompt antibiotic treatment adjusted to renal function and culture results. Cyst infection requires antibiotics that penetrate the cyst (eg macrolides, ciprofloxacin, trimethoprim etc). A prolonged course of antibiotics may be required. Surgical intervention is occasionally indicated. In the presence of recurrent infection, it would be prudent to consider long-term prophylactic antibiotics. ${ }^{15}$

\section{Cyst haemorrhage and haematuria}

Conservative management with bed rest, hydration and analgesia is the mainstay of

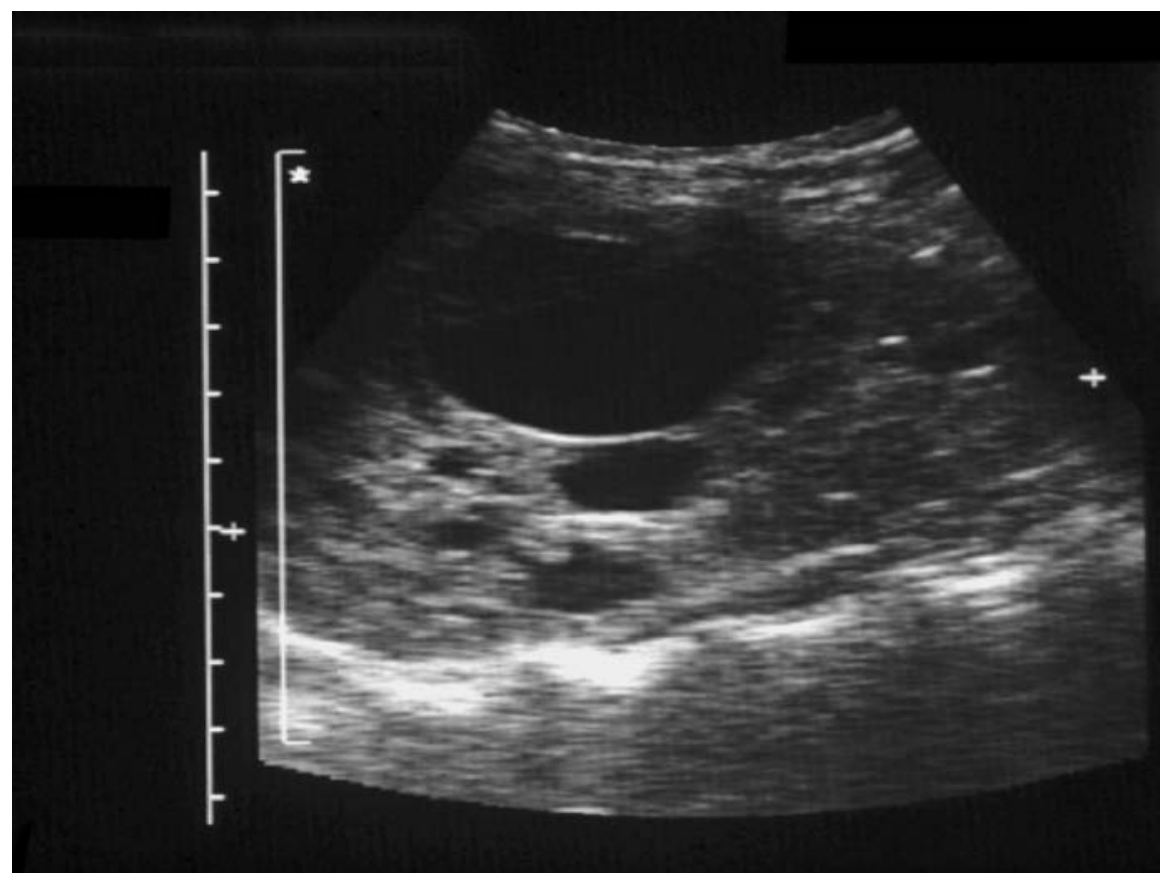

Fig 2. Ultrasound scan showing multiple cysts within the kidney.

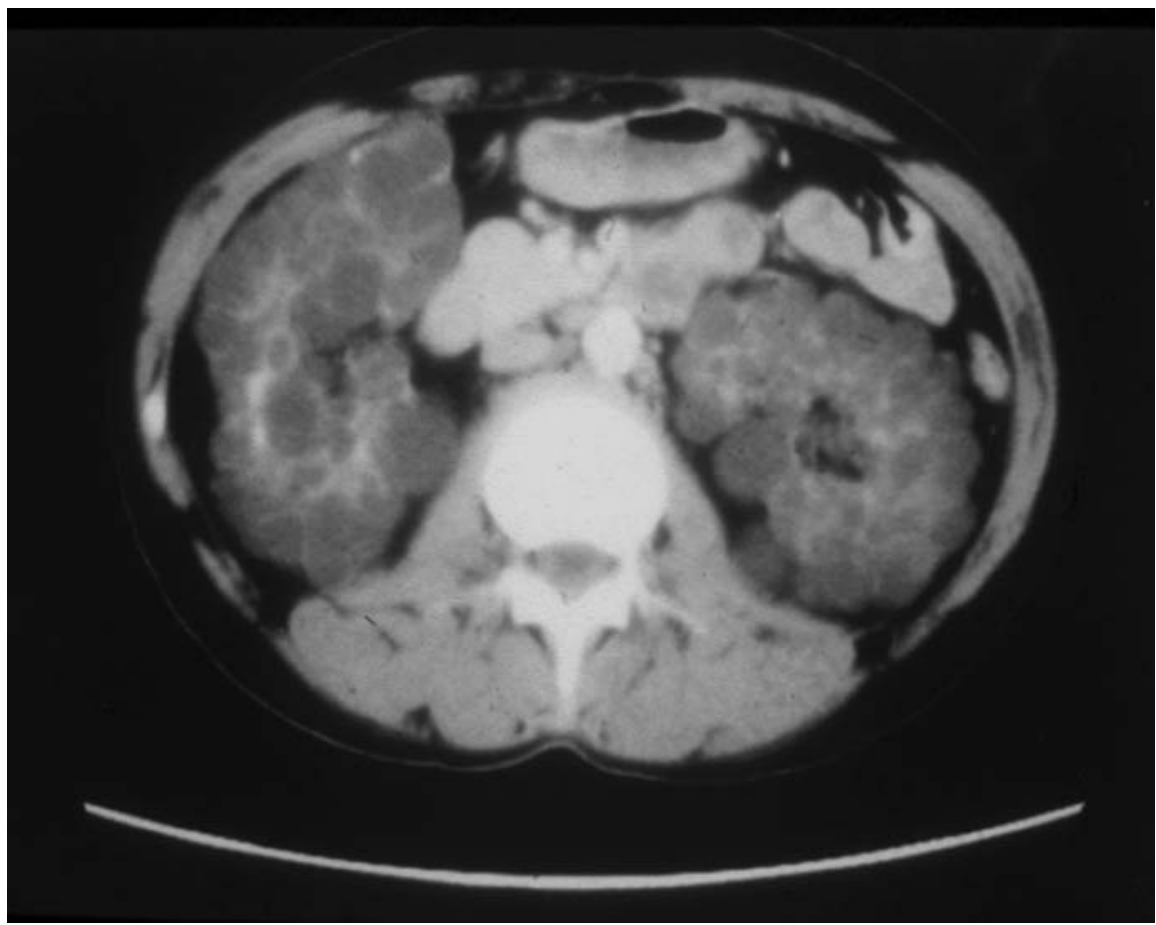

Fig 3. Computed tomography scan showing bilateral enlarged polycystic kidneys. therapy for cyst haemorrhage and haematuria as bleeding is normally self-limiting and resolves within days. ${ }^{15}$ Further investigation to exclude malignancy may be considered if episodes are frequent and/or prolonged (especially in patients over 50 years old) or if other systemic symptoms are present. Rarely, haemodynamically significant bleeding can occur, requiring hospital admission, transfusion and computed tomography with or without angiography. Persistent haemorrhage may require segmental arterial embolisation (coils) or nephrectomy.

\section{Nephrolithiasis}

Renal stones normally resolve with conservative measures: hydration, treatment of infection and analgesia. More complicated cases should be referred for extracorporeal shockwave lithotripsy and percutaneous nephrostolithotomy. ${ }^{3}$

\section{Massive polycystic kidneys and large renal cysts}

Cyst reduction by alcohol sclerosis, percutaneous drainage or laparoscopic deroofing are options. Laparoscopic renal denervation can be considered in rare cases of uncontrolled pain. ${ }^{3}$ Nephrectomy

\section{Key Points}

Autosomal dominant polycystic kidney disease is a common genetic disease due to mutations in two genes, PKD1 and PKD2

Patients may present with or develop significant extrarenal manifestations

The main aim of therapy is directed at blood pressure control, prompt treatment of infections, addressing cardiovascular risk and treating the complications of chronic kidney disease

Renal ultrasound remains the major screening method

Unified diagnostic criteria for at-risk individuals have been published

KEY WORDS: chronic kidney disease, genetic renal disease, intracerebral aneurysms, polycystic kidney disease, screening 
is indicated for massive kidneys (occupying surgical space) in a transplant work-up, severe resistant recurrent infection, severe pain or malignancy.

\section{Liver cysts}

Infection will require antibiotics with good cyst penetration, often given for a long course. If the infection does not settle, patients may require cyst drainage or hepatectomy in rare cases. If there are problems with satiety that impact on nutrition or severe discomfort, a surgical debulking procedure could be considered. ${ }^{3}$

\section{Table 2. Investigations.}

\section{Laboratory studies}

Serum chemistry profile, calcium and phosphate for CKD staging and monitoring Urinalysis and culture:

- Microscopic haematuria $25 \%$, overt proteinuria $18 \%$

- Nephrotic range proteinuria is rare and should prompt further investigation for concomitant glomerulonephritis

- Sterile pyuria is common

Mutation testing:

- Mutation analysis for PKD1 and PKD2 has a detection rate of up to $89 \%$, but with a high level of unclassified variants (26-37\%) and a high rate of polymorphic variants for PKD $1^{11,12}$

- Mosaicism in PKD1 and a low frequency of gene deletions have recently been reported $^{13,14}$

\section{Imaging studies}

Ultrasound:

Diagnostic criteria for ADPKD with positive family history:8,9

- $\geqslant 3$ cysts in one or both kidneys in patients aged 15-39 years

- $\geqslant 2$ cysts in each kidney in patients aged 40-59 years

- $\geqslant 4$ cysts in each kidney in patients aged $\geqslant 60$ years

In sporadic cases or those with atypical features, mutation testing may be required for a positive diagnosis

$\mathrm{CT}$ and MR imaging:

- Enhanced sensitivity for cyst detection, but not routinely used for diagnostic purposes

- Useful in the differential diagnosis of cyst haemorrhage, renal cell carcinoma, simple cysts and renal stones

- Serial MRI can be used to monitor the rate of kidney volume expansion ${ }^{10}$

MR angiography:

- Preferred imaging technique for diagnosing $\mathrm{ICA}^{6}$

- Screening is recommended in patients with:

-family history of ICA

-symptoms of ICA

-a high-risk occupation eg airline pilot

-previous ICA

- Routine screening of asymptomatic ADPKD patients is not recommended

ADPKD = autosomal dominant polycystic kidney disease; $C K D=$ chronic kidney disease; $\mathrm{CT}$ = computed tomography; ICA = intracerebral aneurysm; MRI = magnetic resonance.
If an aneurysm larger than $7 \mathrm{~mm}$ in diameter is detected, follow-up surveillance scans will be required. ${ }^{17}$ Control of hypertension and hyperlipidaemia, and termination of smoking are advised. The risk of a new aneurysm developing after an initial negative study is about $2.6 \%$ at 10 years in patients with a positive family history of ICA. ${ }^{18}$

\section{Chronic kidney disease and renal replacement therapy}

Both peritoneal dialysis and haemodialysis can be used in ADPKD patients. Transplantation is the treatment of choice but pre-emptive nephrectomy may be required if kidneys are massively enlarged. There is no difference in patient or graft survival in ADPKD patients compared with other ESRF populations. ${ }^{15}$ However a threefold increased risk of developing new-onset diabetes after transplantation in ADPKD has recently been reported. ${ }^{19}$

\section{Family screening}

Patients should be advised to inform atrisk blood relatives of the diagnosis and the need for screening. At consultation, the inheritance pattern should be explained, with advice on methods of presymptomatic diagnosis, including the possibility of prenatal diagnosis. Each atrisk individual should be informed of the consequences of diagnostic screening, including the possibility of false-negative ultrasound tests in younger patients.

\section{Making a decision to diagnose}

\section{Pros}

- early management of complications

- family planning

- absence of ADPKD reassures patient.

\section{Cons}

- psychological consequences as no specific treatment or cure is available at present

- insurance and employment issues.

Family members who opt not to have screening should be advised to have 


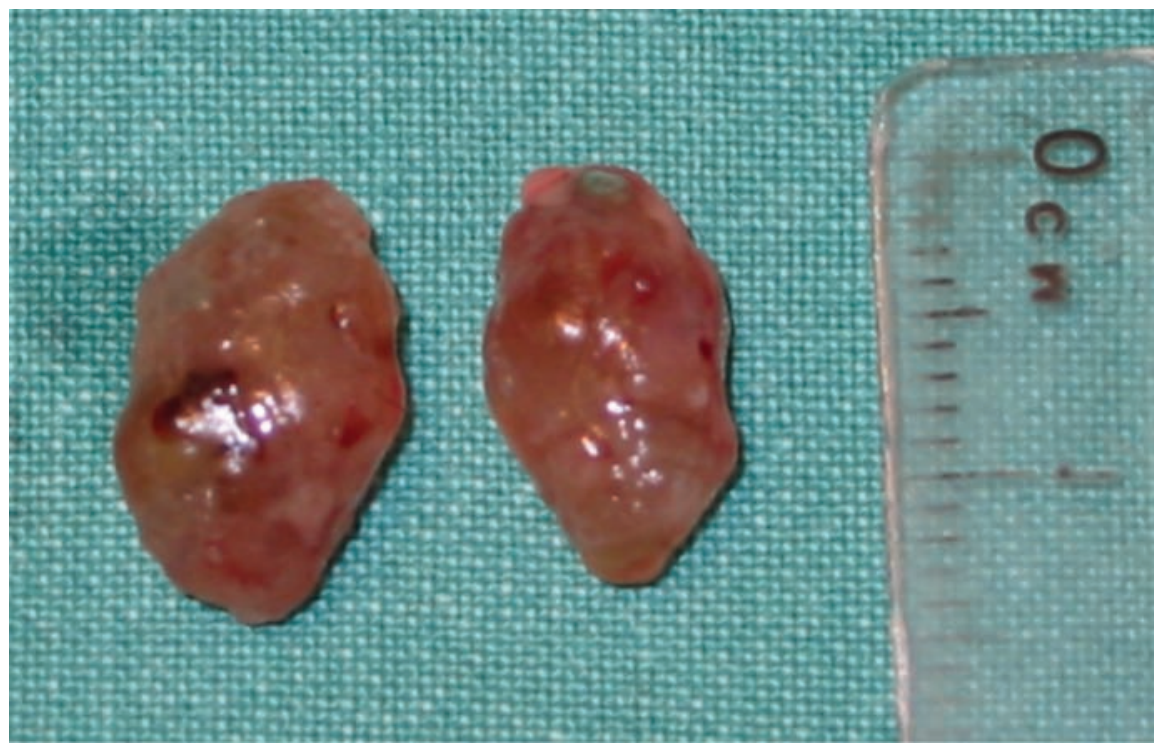

Fig 4. Pathological specimen of PKD2 knockout mouse kidneys showing kidney enlargement due to multiple cysts.

yearly checks on renal function, blood pressure and urinalysis, and to inform their general practitioner of their risk of developing ADPKD.

\section{Pregnancy}

There should be preconception counselling and referral to specialist renal and obstetric services. Prenatal diagnosis by chorionic villous sampling can be done at the ninth week of pregnancy. This is not performed routinely due to high intrafamilial phenotypic variability. Prenatal screening may be considered in families with one severely affected child (early-onset PKD) who have a calculated $45 \%$ recurrence risk for following pregnancies. ${ }^{20}$

The risk of worsening renal function and complications in pregnancy are higher in the presence of moderate-tosevere renal impairment, proteinuria and hypertension. Hypertensive complications, including new and worsening hypertension, have been reported in $25 \%$ of patients, with pre-eclampsia in $11 \%$.

\section{Future prospects}

Several promising candidate drugs have commenced phase 3 clinical trials, based largely on their efficacy in rodent models of PKD:

- A multicentre trial of tolvaptan, a vasopressin type 2 receptor antagonist, commenced in 2007. ${ }^{21}$

- Octreotide, a somatostatin analogue, ${ }^{22}$ has been shown to inhibit kidney growth in a small phase 2 crossover study.

- Rapamycin, ${ }^{23}$ phase 3 clinical trials of sirolimus and everolimus (a derivative of rapamycin) have commenced.

\section{References}

1 Ong AC, Harris PC. Molecular pathogenesis of ADPKD: the polycystin complex gets complex. Kidney Int 2005;67: 1234-47.

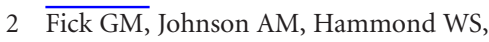
Gabow PA. Causes of death in autosomal dominant polycystic kidney disease. $\mathrm{JAm}$ Soc Nephrol 1995;5:2048-56.

3 Torres VE, Harris PC, Pirson Y. Autosomal dominant polycystic kidney disease. Lancet 2007;369:1287-301.

4 Vora N, Perrone R, Bianchi DW. Reproductive issues for adults with autosomal dominant polycystic kidney disease. Am J Kidney Dis 2008;51:307-18.

5 Cobben JM, Breuning MH, Schoots C, ten Kate LP, Zerres K. Congenital hepatic fibrosis in autosomal-dominant polycystic kidney disease. Kidney Int 1990;38:880-5.

6 Pirson Y, Chauveau D, Torres V. Management of cerebral aneurysms in autosomal dominant polycystic kidney disease. J Am Soc Nephrol 2002;13:269-76.
7 Torra R, Nicolau C, Badenas C et al. Ultrasonographic study of pancreatic cysts in autosomal dominant polycystic kidney disease. Clin Nephrol 1997;47:19-22.

8 Pei Y, Obaji J, Dupuis A et al. Unified criteria for ultrasonographic diagnosis of ADPKD. J Am Soc Nephrol 2008;20:205-12.

9 Ravine D, Gibson RN, Walker RG et al. Evaluation of ultrasonographic diagnostic criteria for autosomal dominant polycystic kidney disease 1. Lancet 1994;343:824-7.

10 Grantham JJ, Torres VE, Chapman AB et al. Volume progression in polycystic kidney disease. N Engl J Med 2006;354:2122-30.

11 Rossetti $\overline{\mathrm{S} \text {, Consugar MB, Chapman } \mathrm{AB}}$ et al. Comprehensive molecular diagnostics in autosomal dominant polycystic kidney disease. J Am Soc Nephrol 2007; 18:2143-60.

12 Garcia-Gonzalez MA, Jones JG, Allen SK et al. Evaluating the clinical utility of a molecular genetic test for polycystic kidney disease. Mol Genet Metab 2007;92:160-7.

13 Connor A, Lunt PW, Dolling C et al. Mosaicism in autosomal dominant polycystic kidney disease revealed by genetic testing to enable living related renal transplantation. Am J Transplant 2008; 8:232-7.

14 Consugar MB, Wong WC, Lundquist PA et al. Characterization of large rearrangements in autosomal dominant polycystic kidney disease and the PKD1/TSC2 contiguous gene syndrome. Kidney Int 2008; 74:1468-79.

15 Grantham JJ. Clinical practice. Autosomal dominant polycystic kidney disease. $N$ Engl J Med 2008;359:1477-85.

16 Gibbs GF, Huston J 3rd, Qian Q et al. Follow-up of intracranial aneurysms in autosomal-dominant polycystic kidney disease. Kidney Int 2004;65:1621-7.

17 Wiebers DO, Whisnant JP, Huston J 3rd et al. Unruptured intracranial aneurysms: natural history, clinical outcome, and risks of surgical and endovascular treatment. Lancet 2003;362:103-10.

18 Schrier RW, Belz MM, Johnson AM et al. Repeat imaging for intracranial aneurysms in patients with autosomal dominant polycystic kidney disease with initially negative studies: a prospective ten-year follow-up. I Am Soc Nephrol 2004;15:1023-8.

19 Hamer RA, Chow CL, Ong AC, McKane WS. Polycystic kidney disease is a risk factor for new-onset diabetes after transplantation. Transplantation 2007; 83:36-40.

20 Zerres K, Rudnik-Schöneborn S, Deget F. Childhood onset autosomal dominant polycystic kidney disease in sibs: clinical picture and recurrence risk. German Working Group on Paediatric Nephrology (Arbeitsgemeinschaft für Pädiatrische Nephrologie). J Med Genet 1993;30:583-8. 
21 Wang X, Gattone V 2nd, Harris PC, Torres VE. Effectiveness of vasopressin V2 receptor antagonists OPC-31260 and OPC-41061 on polycystic kidney disease development in the PCK rat. J Am Soc Nephrol 2005; 16:846-51. Grantham JJ. Does extended-release somatostatin slow the growth of renal cysts in autosomal-dominant polycystic kidney disease? Nat Clin Pract Nephrol 2006;2:66-7.

23 R, Serra AL, Le Hir M et al. Inhibition of mTOR with sirolimus slows disease progression in Han:SPRD rats with autosomal dominant polycystic kidney disease (ADPKD). Nephrol Dial Transplant 2006;21:598-604.
Address for correspondence: Professor ACM Ong, Academic Unit of Nephrology, The Henry Wellcome Laboratories for Medical Research, University of Sheffield Medical School, Beech Hill Road, Sheffield S10 2RX.

Email: A.Ong@sheffield.ac.uk

\section{NCC-CC GUIDELINES}

\section{Chronic kidney disease}

\section{National clinical guideline for early identification and management in adults in primary and secondary care}

Chronic kidney disease (CKD) is of growing importance in the UK. The NHS is increasingly focusing on prevention and on the early detection and treatment of this potentially progressive disease, while the prevalence of risk factors for CKD, such as diabetes, obesity and hypertension, is rising.

In commissioning this guideline, the Department of Health was clear that its focus should be early detection and management. This is the area where the guideline can deliver its greatest potential benefit, by delaying progression of disease and therefore reducing the need for dialysis or transplant. The key recommendations singled out in the guideline reflect this emphasis. They present clear criteria for testing for CKD, for suspecting progressive CKD, and for referring people for specialist assessment, all of which will be useful in primary care. Recommendations are also provided on starting treatment once proteinuria has been assessed. As well as providing a comprehensive guide to the identification and management of patients with CKD for general practitioners and general physicians, the guideline will also be relevant to nephrologists, diabetologists, nurses, clinical biochemists and other health professionals involved in the care of patients with CKD.

Healthcare professionals in primary care are taking on an increasing role in the management of CKD; and this guideline provides a single useful and accessible reference promoting a consistent high quality of care, and an improved quality of life, for longer, for people with CKD.
Royal College of Physicians setting higher medical standards

The National Collaborating Centre for Chronic Conditions

This guideline is part of a series commissioned by NICE which aims to ensure that standards of care throughout England and Wales are uniformly high.

Developed by the National Collaborating Centre for Chronic Conditions at the Royal College of Physicians

Published SEPTEMBER 2008

ISBN: 978-1-86016-340-1

Price: $£ 30.00$ UK, $£ 33.00$ overseas (prices include postage and packing)

Please quote the reference Clinical Medicine when making your order 